\title{
$p$ TH MEAN INTEGRABILITY AND ALMOST SURE ASYMPTOTIC STABILITY OF SOLUTIONS OF ITÔ-VOLTERRA EQUATIONS
}

\author{
JOHN A.D. APPLEBY
}

\begin{abstract}
This paper studies the pathwise asymptotic stability and integrability of the zero solution of a finitedimensional Itô-Volterra equation. Under Lipschitz conditions on the state-dependent functions, and with continuity and integrability required of the kernels, it is shown that any solution which is $p$ th mean integrable for some $p \geq 2$ is $p$ th mean-asymptotically stable, and also $p$ th mean integrable and asymptotically stable, almost surely. If there is no delaydependent term in the volatility, the same result can be shown for $p \geq 1$. Examples which illustrate the usefulness of these results are presented, and extensions to other classes of functional differential equations are discussed.
\end{abstract}

1. Introduction. Much research in recent years has focused on the almost sure exponential asymptotic stability of solutions of stochastic differential equations and stochastic delay differential equations with bounded delay, with several recent monographs appearing by Mao $[\mathbf{1 1}$, 12]. However, less attention has been devoted to the almost sure exponential asymptotic stability of Itô-Volterra equations, where the delay is unbounded. It has been shown in Appleby and Reynolds [3] for nonlinear scalar time-homogeneous Itô-Volterra equations of the form

(1) $d X(t)=\left(f(X(t))+\int_{0}^{t} k(t-s) g(X(s)) d s\right) d t+h(X(t)) d B(t)$

for which the kernel $k$ is strictly positive and satisfies a nonexponential asymptotic condition, the solution satisfies

$$
\limsup _{t \rightarrow \infty}|X(t)| e^{\varepsilon t}=\infty, \quad \text { a.e. on } A
$$

1991 AMS Mathematics Subject Classification. Primary 34K20, Secondary $60 \mathrm{H} 10$.

Key words and phrases. Almost sure asymptotic stability, pth mean asymptotic stability, Itô-Volterra equations, stochastic functional differential equations, Volterra equations.

Received by the editors on February 3, 2003, and in revised form on August 4, 2003.

Copyright (C)2003 Rocky Mountain Mathematics Consortium 
for every $\varepsilon>0$, where $A=\{\omega \in \Omega: X(t, \omega) \rightarrow 0$ as $t \rightarrow \infty\}$. This result is a stochastic analogue of the deterministic case which was presented in this journal in [2]. These results are instances of a more general stability phenomenon exhibited by equations with unbounded delay. For deterministic Volterra integrodifferential equations, Murakami has shown in $[\mathbf{1 6}, \mathbf{1 7}]$ that if all solutions of the finite dimensional equation

$$
x^{\prime}(t)=A x(t)+\int_{0}^{t} K(t-s) x(s) d s
$$

converge exponentially fast to the zero solution, and the entries of the integrable kernel $K$ do not change sign on $\mathbf{R}^{+}$, then

$$
\int_{0}^{\infty}\|K(s)\| e^{\gamma s} d s<\infty, \quad \text { for some } \gamma>0
$$

Therefore, exponential stability cannot always be guaranteed if the kernel does not vanish exponentially fast, in some sense. Thus, the question of asymptotic stability of functional differential equations with unbounded delay is one of independent interest from the exponential stability, not only for deterministic equations, but potentially for stochastic ones also. In this paper attempts are made to develop some sufficient conditions under which almost sure stability can be established.

For stochastic differential equations and stochastic functional differential equations, it is known that almost sure exponential asymptotic stability is often implied by exponential stability in $p$ th mean, see Arnold, Oeljeklaus and Pardoux [4] for linear SDEs and Mao [12] for nonlinear SDEs, and SFDEs with bounded delay. In [13], mean square and almost sure exponential asymptotic stability are established for an Itô-Volterra equation which has an exponential decay criterion required on the kernels. Hausmann [8] and Zabczyk [18] provide a connection between the square integrability of the mild solution of a linear bounded delay equation of Lurie type, and the almost sure exponential stability of the strong solution of the stochastic delay equation.

The main result presented is motivated by the above theory for stochastic equations and also by the fact that, if the solution of the linear deterministic Volterra equation (2) is integrable, then its solution tends to zero as $t \rightarrow \infty$. We show that this result for deterministic 
Volterra equations can be extended to Itô-Volterra equations with solution $X$, if the integrability condition is amended to

$$
\mathbf{E}\left[\|X(t)\|^{p}\right] \in L^{1}\left(\mathbf{R}^{+}\right),
$$

for some $p \geq 2$. Then, not only does the solution satisfy

$$
\lim _{t \rightarrow \infty} \mathbf{E}\left[\|X(t)\|_{2}^{p}\right]=0,
$$

but the solution is also almost surely asymptotically stable; that is,

(6)

$\lim _{t \rightarrow \infty} X(t, \omega)=0$ for almost all outcomes $\omega$ in the sample space $\Omega$.

Moreover, $X \in L^{p}\left(\mathbf{R}^{+}\right)$almost surely. This result is the subject of Theorem 4. The result is sharpened for some special cases in Theorem 5. The proofs are adaptations of results from $[8]$ and Mao [12, Chapter 4].

These adaptations are necessitated as the hypothesis (4) does not provide a pointwise estimate on the decay rate of $t \mapsto \mathbf{E}\left[\|X(t)\|^{p}\right]$. Indeed, it may be that such a decay rate does not exist. In particular, not only can almost sure exponential decay of solutions not be guaranteed but, as we later establish, exponential decay of solutions in $p$ th mean cannot be guaranteed either.

The moment asymptotic stability and integrability of Itô-Volterra equations has received the attention of several authors, among which we highlight the work of Kolmanovskii and Myskis [10] and Mizel and Trutzer [15]. Hence, Theorem 4 has the benefit of connecting the $p$ th mean stability to the almost sure stability. It also improves results obtained by the author in [1], in which both (4) and (5) are required to prove (6). We show by an example, that because the $p$ th mean integrability only is required, one can lift some of the restrictions placed on Liapunov functionals in order to obtain asymptotic stability, so the result is of additional use in obtaining a.s. asymptotic stability. We also show Theorem 5 enables us to compare the asymptotic stability of a special, but much studied, linear scalar Volterra integrodifferential equation and its stochastic counterpart. Theorem 5 provides sharper results on almost sure stability than can be obtained by using the $p$ th mean integrability, for $p>1$, to prove a.s. asymptotic stability. 
The organization of this presentation is as follows. Section 2 describes in precise terms the problem to be studied, introduces terminology and known results, and proves (or states) preparatory lemmata. Section 3 concerns the proof of Theorems 4 and 5 . Section 4 is devoted to examples which highlight the results of Theorems 4 and 5 . The concluding Section 5 contains a discussion on the extension of the methods of the paper to cover the asymptotic stability of nonconvolution and nonautonomous Itô-Volterra equations and more general stochastic functional differential equations with infinite delay.

2. Statement of the problem and preliminary results. We first establish some standard notation. As usual, let $x \vee y$ denote the maximum of $x, y \in \mathbf{R}$, and $x \wedge y$ their minimum. Let $d, m$ and $r$ be positive integers. Denote by $C\left(\mathbf{R}^{d} ; \mathbf{R}^{m}\right)$ the space of continuous functions from $\mathbf{R}^{d}$ to $\mathbf{R}^{m}$, and $C\left(\mathbf{R}^{d} ; \mathbf{R}^{d} \times \mathbf{R}^{r}\right)$ the space of continuous functions from $\mathbf{R}^{d}$ to $\mathbf{R}^{d} \times \mathbf{R}^{r}$. We identify the space of real valued $d \times m$ matrices, denoted $M_{d, m}(\mathbf{R})$, with $\mathbf{R}^{d} \times \mathbf{R}^{m}$. Let $C\left(\mathbf{R}^{+} ; M_{d, m}(\mathbf{R})\right)$ stand for all continuous $d \times m$ matrix-valued functions with domain $\mathbf{R}^{+}$. For $x, y \in \mathbf{R}^{d}$, we denote the (standard) inner product of $x$ and $y$ by $\langle x, y\rangle$. Let $\|x\|_{2}$ stand for the Euclidean norm of $x \in \mathbf{R}^{d}$. For $A \in M_{d, m}(\mathbf{R})$ denote $\|A\|_{2}$ to be the operator norm of $A$, with $\|A\|_{2}=\max _{\|x\|_{2}=1}\|A x\|$. If $A=\left(a_{i j}\right) \in M_{d, m}(\mathbf{R})$, we denote its Frobenius norm by $\|A\|_{F(d, m)}$ :

$$
\|A\|_{F(d, m)}^{2}=\sum_{i=1}^{d} \sum_{j=1}^{m} a_{i j}^{2} .
$$

Observe, for all $A \in M_{d, m}(\mathbf{R})$ and all $B \in M_{m, r}(\mathbf{R})$, that

$$
\|A B\|_{F(d, r)} \leq \sqrt{m}\|A\|_{F(d, m)}\|B\|_{F(m, r)} .
$$

Denote by $L^{1}\left(\mathbf{R}^{+}\right)$the space of all measurable real valued functions integrable on $\mathbf{R}^{+}$. We say that $K(t) \in M_{d, m}(\mathbf{R})$ is in $L^{1}\left(\mathbf{R}^{+}\right)$if each of its entries is in $L^{1}\left(\mathbf{R}^{+}\right)$. For such a function $K$, we write

$$
\|K\|_{F(d, m)}^{L^{1}\left(\mathbf{R}^{+}\right)}=\int_{0}^{\infty}\|K(t)\|_{F(d, m)} d t, \quad\|K\|_{2}^{L^{1}\left(\mathbf{R}^{+}\right)}=\int_{0}^{\infty}\|K(t)\|_{2} d t .
$$

We denote the convolution of the functions $x_{1}, x_{2} \in C\left(\mathbf{R}^{+} ; \mathbf{R}\right)$ by $x_{1} * x_{2}$. 
Let $f_{1} \in C\left(\mathbf{R}^{d} ; \mathbf{R}^{d}\right), g_{1} \in C\left(\mathbf{R}^{d} ; \mathbf{R}^{n}\right), f_{2} \in C\left(\mathbf{R}^{d} ; \mathbf{R}^{d} \times \mathbf{R}^{r}\right)$, and $g_{2} \in C\left(\mathbf{R}^{d} ; \mathbf{R}^{m} \times \mathbf{R}^{r}\right)$, while

$K_{1} \in C\left(\mathbf{R}^{+} ; M_{d, n}(\mathbf{R})\right) \cap L^{1}\left(\mathbf{R}^{+}\right), \quad K_{2} \in C\left(\mathbf{R}^{+} ; M_{d, m}(\mathbf{R})\right) \cap L^{1}\left(\mathbf{R}^{+}\right)$.

Further suppose that $f_{1}, f_{2}, g_{1}, g_{2}$ satisfy global Lipschitz conditions. For definiteness, there exists some $\lambda>0$ such that

$$
\begin{aligned}
\left\|f_{1}(x)-f_{1}(y)\right\|_{2} & \vee\left\|f_{2}(x)-f_{2}(y)\right\|_{F(d, r)} \\
& \vee\left\|g_{1}(x)-g_{1}(y)\right\|_{2} \\
& \vee\left\|g_{2}(x)-g_{2}(y)\right\|_{F(m, r)} \leq \lambda\|x-y\|_{2}
\end{aligned}
$$

for all $x, y \in \mathbf{R}^{d}$. We also assume

$$
\begin{gathered}
f_{1}(0)=0 \in \mathbf{R}^{d}, \quad f_{2}(0)=0 \in \mathbf{R}^{d} \times \mathbf{R}^{r}, \\
g_{1}(0)=0 \in \mathbf{R}^{d} \times \mathbf{R}^{n}, \quad g_{2}(0)=0 \in \mathbf{R}^{m} \times \mathbf{R}^{r} .
\end{gathered}
$$

(9), (10) imply that $f_{1}, f_{2}, g_{1}, g_{2}$ are linearly bounded.

Let $(\Omega, \mathcal{F}, \mathbf{P})$ be a probability space and $B=\left\{B(t), \mathcal{F}_{t}^{B} ; 0 \leq t<\infty\right\}$ an $r$-dimensional Brownian motion on it. Let $\xi$ be a random vector in $\mathbf{R}^{d}$, independent of $B$ with $\mathbf{E}\left[\|\xi\|^{p \vee 2}\right]<\infty$, where $p \geq 1$. Then there exists a filtration $\left(\mathcal{F}_{t}\right)_{t \geq 0}$ so that $\left\{B(t), \mathcal{F}_{t} ; 0 \leq t<\infty\right\}$ is an $r$-dimensional Brownian motion. Under the above hypotheses, there exists a continuous adapted process $X=\left\{X(t) ; \mathcal{F}_{t} ; 0 \leq t<\infty\right\}$ which is a strong solution of the Itô-Volterra equation

$$
\begin{aligned}
d X(t)= & \left(f_{1}(X(t))+\int_{0}^{t} K_{1}(t-s) g_{1}(X(s)) d s\right) d t \\
& +\left(f_{2}(X(t))+\int_{0}^{t} K_{2}(t-s) g_{2}(X(s)) d s\right) d B(t)
\end{aligned}
$$

relative to $B$ with initial condition $\xi$, see Berger and Mizel [5] or Itô and Nisio [9]. The hypotheses also ensure that if $\xi=0$ a.s., then the solution of (11) is $X(t)=0$ for all $t \geq 0$ a.s. This is called the zero solution to (11).

The purpose of the paper is the following: to establish conditions under which there exists $\Omega^{*} \subseteq \Omega$ with $\mathbf{P}\left[\Omega^{*}\right]=1$ such that, for all $\omega \in \Omega^{*}$

$$
\lim _{t \rightarrow \infty} X(t, \omega)=0
$$


whatever the value of the initial condition $X(0)=\xi$. If (12) holds we say that the solution of (11) is almost surely (globally) asymptotically stable.

We will make use of the following result on the continuity of the moments of the solution, proved in $[\mathbf{1}]$.

Lemma 1. Under the forgoing hypotheses, we have

(i) $\mathbf{E}\left[\|X(0)\|^{p}\right]<\infty$,

(ii) For $0 \leq T<\infty, \mathbf{E}\left[\sup _{0 \leq t \leq T}\|X(t)\|^{p}\right] \leq K_{p}(T)<\infty$.

(iii) The mapping $t \mapsto \mathbf{E}\left[\|X(t)\|^{p}\right]$ is continuous on $\mathbf{R}^{+}$.

For $x \in C\left(\mathbf{R}^{+} ; \mathbf{R}^{d}\right)$, define $x_{t}=\{x(s): 0 \leq s \leq t\}$. Define the functionals $Y_{1}: \mathbf{R}^{+} \times C\left(\mathbf{R}^{+} ; \mathbf{R}^{d}\right) \rightarrow \mathbf{R}^{d}, Y_{2}: \mathbf{R}^{+} \times C\left(\mathbf{R}^{+} ; \mathbf{R}^{d}\right) \rightarrow$ $\mathbf{R}^{d} \times \mathbf{R}^{r}$, so that

$$
\begin{aligned}
& Y_{1}\left(t, x_{t}\right)=f_{1}(x(t))+\int_{0}^{t} K_{1}(t-s) g_{1}(x(s)) d s, \\
& Y_{2}\left(t, x_{t}\right)=f_{2}(x(t))+\int_{0}^{t} K_{2}(t-s) g_{2}(x(s)) d s .
\end{aligned}
$$

Then it is possible to write the equation (11) as

$$
X(t)=X(0)+\int_{0}^{t} Y_{1}\left(s, X_{s}\right) d s+\int_{0}^{t} Y_{2}\left(s, X_{s}\right) d B(s) .
$$

We use the following estimates on the functionals $Y_{1}, Y_{2}$ throughout the paper, which are established in $[\mathbf{1}]$.

Lemma 2. If $x \in C\left(\mathbf{R}^{+} ; \mathbf{R}^{d}\right)$, then the functionals defined by (13), (14) satisfy:

$$
\left\|Y_{1}\left(t, x_{t}\right)\right\|_{2} \leq \lambda\|x(t)\|_{2}+\lambda\left(\left\|K_{1}\right\|_{2} *\|x\|_{2}\right)(t),
$$

and

$$
\begin{aligned}
& \left\|Y_{2}\left(t, x_{t}\right)\right\|_{F(d, r)}^{2} \\
& \quad \leq 2 \lambda^{2}\|x(t)\|_{2}^{2}+2 \lambda^{2} m\left\|K_{2}\right\|_{F(d, m)}^{L^{1}\left(\mathbf{R}^{+}\right)}\left(\left\|K_{2}\right\|_{F(d, m)} *\|x\|_{2}^{2}\right)(t),
\end{aligned}
$$


for $t \geq 0$.

The following result will also be required.

Lemma 3. Suppose $x: \mathbf{R}^{+} \rightarrow \mathbf{R}^{+}$is a continuous, integrable function, and $\mu>0$ is any fixed constant. Then, there exists a sequence $\left\{a_{n}\right\}_{n=0}^{\infty}$ satisfying

$$
a_{0}=0, \quad 0<a_{n+1}-a_{n}<\mu \quad \text { for all } n \in N, \quad \lim _{n \rightarrow \infty} a_{n}=\infty,
$$

together with

$$
\sum_{n=0}^{\infty} x\left(a_{n}\right)<\infty .
$$

Proof. Construct $\left\{a_{n}\right\}_{n=0}^{\infty}$ recursively as follows: let $a_{0}=0$, and for $n \in \mathbf{N}$

$a_{n+1}=\inf \left\{t \in\left[a_{n}+\mu / 2, a_{n}+3 \mu / 4\right]: x(t)=\min _{a_{n}+\mu / 2 \leq \tau \leq a_{n}+3 \mu / 4} x(\tau)\right\}$.

The existence of such a sequence can be proved by induction on $n$, taking note that $x$ is continuous on the compact interval $\left[a_{n}+\mu / 2, a_{n}+\right.$ $3 \mu / 4]$, and hence attains its minimum. By construction, we have

$$
a_{n+1}-a_{n}>\frac{\mu}{4}>0,
$$

and $a_{n+1}-a_{n} \leq 3 \mu / 4<\mu$, so $\left\{a_{n}\right\}_{n=0}^{\infty}$ satisfies (18). To prove (19), note that $x\left(a_{n+1}\right) \leq x(t)$ for $a_{n}+\mu / 2 \leq t \leq a_{n}+3 \mu / 4$, so by integrating both sides of this inequality over $\left[a_{n}+\mu / 2, a_{n}+3 \mu / 4\right]$, using the nonnegativity of $x(\cdot)$ and $a_{n}+3 \mu / 4<a_{n+1}+\mu / 2$, which follows from (20), we get

$$
\frac{1}{4} \mu x\left(a_{n+1}\right) \leq \int_{a_{n}+\mu / 2}^{a_{n}+3 \mu / 4} x(t) d t \leq \int_{a_{n}+\mu / 2}^{a_{n+1}+\mu / 2} x(t) d t .
$$

Summing both sides of this inequality establishes (19). 
If $\mathbf{E}\left[\|X(t)\|^{p}\right] \in L^{1}\left(\mathbf{R}^{+}\right)$for some $p>0$, then $x(t)=\mathbf{E}\|X(t)\|^{p}$ satisfies the conditions of Lemma 3 , by Lemma 1 (i), (iii). Thus, for every $\mu>0$, there exists a sequence $\left\{a_{n}\right\}_{n=0}^{\infty}$ satisfying (18) and for which

$$
\sum_{n=0}^{\infty} \mathbf{E}\left[\left\|X\left(a_{n}\right)\right\|^{p}\right]<\infty .
$$

Recall by the Burkholder-Davis-Gundy inequality that, for every $p \geq 0$, there exists a constant $C_{p}>0$ such that

$$
\mathbf{E}\left[\max _{a \leq t \leq b}\left\|\int_{a}^{t} Y(s) d B(s)\right\|^{p}\right] \leq C_{p} \mathbf{E}\left[\left(\int_{a}^{b}\|Y(s)\|_{F(d, r)}^{2} d s\right)^{\frac{p}{2}}\right]
$$

where $C_{p}$ is independent of $0 \leq a \leq b$ and the $M_{d \times r}(\mathbf{R})$-valued $\mathcal{F}_{t^{-}}$ adapted process $Y$. Define $\mu>0$ by

$$
\mu=\left(\frac{1}{3} \lambda\right)^{2}\left(\frac{3}{2 C_{p}}\right)^{2 / p}
$$

and let $\left\{a_{n}\right\}_{n=0}^{\infty}$ be a sequence for which (18) and (21) hold.

3. Proofs. We are now in a position to prove the main results of the paper.

Theorem 4. Suppose that $f_{1}, f_{2}, g_{1}, g_{2}$ satisfy the hypotheses (9), (10) and $K_{1}, K_{2}$ are continuous and integrable functions. Let $X$ be the unique strong solution of (11). If there exists $p \geq 2$ such that $\mathbf{E}\left[\|X(0)\|^{p}\right]<\infty$, and

$$
\int_{0}^{\infty} \mathbf{E}\left[\|X(t)\|^{p}\right] d t<\infty
$$

then each of the following holds:

$$
\begin{gathered}
\lim _{t \rightarrow \infty} \mathbf{E}\left[\|X(t)\|^{p}\right]=0, \\
X(t) \in L^{p}\left(\mathbf{R}^{+}\right), \quad \text { a.s., } \\
\lim _{t \rightarrow \infty} X(t)=0, \quad \text { a.s. }
\end{gathered}
$$


Proof. Let $\mu>0$ be given by (22) and $\left\{a_{n}\right\}_{n=0}^{\infty}$ be a sequence for which (18) and (21) hold. Thus, for each $t \geq 0$, there exists $n \in \mathbf{N}$ such that $a_{n} \leq t<a_{n+1}$. By (18), (13) and (14), we have

$$
X(t)=X\left(a_{n}\right)+\int_{a_{n}}^{t} Y_{1}\left(s, X_{s}\right) d s+\int_{a_{n}}^{t} Y_{2}\left(s, X_{s}\right) d B(s) .
$$

Taking norms on both sides of this equality, using the triangle inequality and the scalar inequality, $(a+b+c)^{p} \leq 3^{p-1}\left(a^{p}+b^{p}+c^{p}\right)$ yields

$$
\begin{gathered}
\|X(t)\|_{2}^{p} \leq 3^{p-1}\left(\left\|X\left(a_{n}\right)\right\|_{2}^{p}+\left\|\int_{a_{n}}^{t} Y_{1}\left(s, X_{s}\right) d s\right\|_{2}^{p}\right. \\
\left.+\left\|\int_{a_{n}}^{t} Y_{2}\left(s, X_{s}\right) d B(s)\right\|_{2}^{p}\right) .
\end{gathered}
$$

Therefore, taking suprema on each side of this inequality, and then taking the expectations of both sides, we get

$$
\begin{aligned}
\mathbf{E}\left[\max _{a_{n} \leq t \leq a_{n+1}}\|X(t)\|_{2}^{p}\right] \leq & 3^{p-1}\left(\mathbf{E}\left[\left\|X\left(a_{n}\right)\right\|_{2}^{p}\right]\right. \\
& +\mathbf{E}\left[\max _{a_{n} \leq t \leq a_{n+1}}\left\|\int_{a_{n}}^{t} Y_{1}\left(s, X_{s}\right) d s\right\|_{2}^{p}\right] \\
& \left.+\mathbf{E}\left[\max _{a_{n} \leq t \leq a_{n+1}}\left\|\int_{a_{n}}^{t} Y_{2}\left(s, X_{s}\right) d B(s)\right\|_{2}^{p}\right]\right) .
\end{aligned}
$$

Let us obtain estimates for the second and third terms on the righthand side of (25). Using (16), we have

$$
\left\|\int_{a_{n}}^{t} Y_{1}\left(s, X_{s}\right) d s\right\|_{2} \leq \int_{a_{n}}^{t} \lambda\|X(s)\|_{2}+\lambda\left(\left\|K_{1}\right\|_{2} *\|X\|_{2}\right)(s) d s,
$$

so by applying Jensen's inequality, and then taking the maximum over $\left[a_{n}, a_{n+1}\right]$, we get

$$
\begin{aligned}
& \max _{a_{n} \leq t \leq a_{n+1}}\left\|\int_{a_{n}}^{t} Y_{1}\left(s, X_{s}\right) d s\right\|_{2}^{p} \\
& \leq \lambda^{p}\left(a_{n+1}-a_{n}\right)^{p-1} \int_{a_{n}}^{a_{n+1}}\left(\|X(s)\|_{2}+\left(\left\|K_{1}\right\|_{2} *\|X\|_{2}\right)(s)\right)^{p} d s .
\end{aligned}
$$


Applying the scalar inequality $(a+b)^{p} \leq 2^{p-1}\left(a^{p}+b^{p}\right)$, and then Hölder's inequality to the integrand in (26) yields

(27)

$$
\begin{aligned}
& \mathbf{E}\left[\max _{a_{n} \leq t \leq a_{n+1}}\left\|\int_{a_{n}}^{t} Y_{1}\left(s, X_{s}\right) d s\right\|_{2}^{p}\right] \\
& \leq \lambda^{p}\left(a_{n+1}-a_{n}\right)^{p-1} 2^{p-1} \\
& \quad \times \int_{a_{n}}^{a_{n+1}} \mathbf{E}\left[\|X(s)\|_{2}^{p}\right]+\left(\left\|K_{1}\right\|_{2}^{L^{1}\left(\mathbf{R}^{+}\right)}\right)^{p-1}\left(\left\|K_{1}\right\|_{2} * \mathbf{E}\left[\|X\|_{2}^{p}\right]\right)(s) d s,
\end{aligned}
$$

after taking expectations across (26). We now seek an estimate on the third term on the right-hand side of (25). Using the Burkholder-DavisGundy inequality, we obtain

$$
\mathbf{E}\left[\max _{a_{n} \leq t \leq a_{n+1}} \|_{2}^{p}\right] \leq C_{p} \mathbf{E}\left[\left(\int_{a_{n}}^{a_{n+1}}\left\|Y_{2}\left(s, X_{s}\right)\right\|_{F(d, r)}^{2} d s\right)^{\frac{p}{2}}\right] .
$$

Define $\Lambda=2 \lambda^{2}\left(1 \vee m\left\|K_{2}\right\|_{F(d, m)}^{L^{1}\left(\mathbf{R}^{+}\right)}\right)$, so using (17), we obtain

$\int_{a_{n}}^{a_{n+1}}\left\|Y_{2}\left(s, X_{s}\right)\right\|_{F(d, r)}^{2} d s \leq \Lambda \int_{a_{n}}^{a_{n+1}}\|X(s)\|_{2}^{2}+\left(\left\|K_{2}\right\|_{F(d, m)} *\|X\|_{2}^{2}\right)(s) d s$.

Using Hölder's inequality successively, together with the elementary inequality $(a+b)^{p / 2} \leq 2^{(p / 2)-1}\left(a^{p / 2}+b^{p / 2}\right)$, leads to the estimate

$$
\begin{aligned}
& \left(\int_{a_{n}}^{a_{n+1}}\left\|Y_{2}\left(s, X_{s}\right)\right\|_{F(d, r)}^{2} d s\right)^{\frac{p}{2}} \\
& \leq \Lambda^{\frac{p}{2}}\left(a_{n+1}-a_{n}\right)^{\left(\frac{p}{2}\right)-1} \int_{a_{n}}^{a_{n+1}}\left(\|X(s)\|_{2}^{2}+\left(\left\|K_{2}\right\|_{F(d, m)} *\|X\|_{2}^{2}\right)(s)\right)^{\frac{p}{2}} d s \\
& \leq \Lambda^{\frac{p}{2}} 2^{\left(\frac{p}{2}\right)-1}\left(a_{n+1}-a_{n}\right)^{\left(\frac{p}{2}\right)-1} \\
& \quad \times \int_{a_{n}}^{a_{n+1}}\|X(s)\|_{2}^{p}+\left(\left\|K_{2}\right\|_{F(d, m)}^{L^{1}\left(\mathbf{R}^{+}\right)}\right)^{\left(\frac{p}{2}\right)-1}\left(\left\|K_{2}\right\|_{F(d, m)} *\|X\|_{2}^{p}\right)(s) d s .
\end{aligned}
$$

Inserting this estimate into (28), we get

$$
\begin{aligned}
& \mathbf{E}\left[\max _{a_{n} \leq t \leq a_{n+1}}\left\|\int_{a_{n}}^{t} Y_{2}\left(s, X_{s}\right) d B(s)\right\|_{2}^{p}\right] \\
& \leq C_{p} \Lambda^{\frac{p}{2}} 2^{(p / 2)-1}\left(a_{n+1}-a_{n}\right)^{\left(\frac{p}{2}\right)-1} \int_{a_{n}}^{a_{n+1}} \mathbf{E}\left[\|X(s)\|_{2}^{p}\right] \\
& \quad+\left(\left\|K_{2}\right\|_{F(d, m)}^{L^{1}\left(\mathbf{R}^{+}\right)}\right)^{\left(\frac{p}{2}\right)-1}\left(\left\|K_{2}\right\|_{F(d, m)} * \mathbf{E}\left[\|X\|_{2}^{p}\right]\right)(s) d s .
\end{aligned}
$$


Using the estimates (27) and (29) in (25), and noting that $a_{n+1}-a_{n}<$ $\mu$, we get

(30)

$$
\begin{aligned}
\mathbf{E}\left[\max _{a_{n} \leq t \leq a_{n+1}}\|X(t)\|_{2}^{p}\right] \leq & c_{1} \mathbf{E}\left[\left\|X\left(a_{n}\right)\right\|_{2}^{p}\right]+c_{2} \int_{a_{n}}^{a_{n+1}} \mathbf{E}\left[\|X(s)\|_{2}^{p}\right] d s \\
& +c_{3} \int_{a_{n}}^{a_{n+1}}\left(\left\|K_{1}\right\|_{2} * \mathbf{E}\left[\|X\|_{2}^{p}\right]\right)(s) d s \\
& +c_{4} \int_{a_{n}}^{a_{n+1}}\left(\left\|K_{2}\right\|_{F(d, m)} * \mathbf{E}\left[\|X\|_{2}^{p}\right]\right)(s) d s
\end{aligned}
$$

once we define $c_{1}-c_{4}$ to be

$$
\begin{aligned}
& c_{1}=3^{p-1}, \\
& c_{2}=\lambda^{p} \mu^{p-1} 2^{p-1}+C_{p} \Lambda^{\frac{p}{2}} 2^{\left(\frac{p}{2}\right)-1} \mu^{\left(\frac{p}{2}\right)-1}, \\
& c_{3}=\lambda^{p} \mu^{p-1} 2^{p-1}\left(\left\|K_{1}\right\|_{2}^{L^{1}\left(\mathbf{R}^{+}\right)}\right)^{p-1}, \\
& c_{4}=C_{p} \Lambda^{\frac{p}{2}} 2^{\frac{p}{2}-1} \mu^{\frac{p}{2}-1}\left(\left\|K_{2}\right\|_{F(d, m)}^{L^{1}\left(\mathbf{R}^{+}\right)}\right)^{\left(\frac{p}{2}\right)-1} .
\end{aligned}
$$

Since $\mathbf{E}\left[\|X\|_{2}^{p}\right] \in L^{1}\left(\mathbf{R}^{+}\right)$, and $K_{1}, K_{2} \in L^{1}\left(\mathbf{R}^{+}\right)$, it follows that $\left\|K_{j}\right\| * \mathbf{E}\left[\|X\|_{2}^{p}\right] \in L^{1}\left(\mathbf{R}^{+}\right)$for $j=1,2$, and the construction of the sequence $\left\{a_{n}\right\}_{n=0}^{\infty}$ in Lemma 3 implies that (21) holds. Hence, summing both sides of (30) yields

$$
\sum_{n=0}^{\infty} \mathbf{E}\left[\max _{a_{n} \leq t \leq a_{n+1}}\|X(t)\|_{2}^{p}\right]<\infty .
$$

This sequence is convergent, so $\mathbf{E}\left[\max _{a_{n} \leq t \leq a_{n+1}}\|X(t)\|_{2}^{p}\right] \rightarrow 0$ as $n \rightarrow \infty$, which implies (24a). To prove (24c), note that (31) also implies

$$
\sum_{n=1}^{\infty} \max _{a_{n} \leq t \leq a_{n+1}}\|X(t)\|_{2}^{p}<\infty, \quad \text { a.s. }
$$

and so

$$
\lim _{n \rightarrow \infty} \max _{a_{n} \leq t \leq a_{n+1}}\|X(t)\|_{2}^{p}=0, \quad \text { a.s. }
$$

Therefore, as $a_{n} \rightarrow \infty$ as $n \rightarrow \infty,\|X(t)\|_{2}^{p} \rightarrow 0$ as $t \rightarrow \infty$, a.s. Turning to the proof of (24b), note that (23) and Fubini's theorem imply 
$\mathbf{E} \int_{0}^{\infty}\|X(s)\|_{2}^{p} d s<\infty$, which guarantees that $\int_{0}^{\infty}\|X(s)\|_{2}^{p} d s<\infty$ a.s.

With some modifications, the proof of Theorem 4 can be adapted to establish the following result, when there is no delay-dependent term in the volatility coefficient.

Theorem 5. Let $f_{1}, f_{2}, g_{1}$ satisfy the hypotheses (9), (10) and let $K_{1}$ be a continuous and integrable function. Let $X$ be the unique strong solution of

$$
\begin{aligned}
& d X(t) \\
& \quad=\left(f_{1}(X(t))+\int_{0}^{t} K_{1}(t-s) g_{1}(X(s)) d s\right) d t+f_{2}(X(t)) d B(t) .
\end{aligned}
$$

Suppose that there exists $p \geq 1$ such that $\mathbf{E}\left[\|X(0)\|^{p \vee 2}\right]<\infty$, and (23) holds. Then each of (24a), (24b), (24c) are true.

Proof. Choosing $\left\{a_{n}\right\}_{n=0}^{\infty}$ so as to satisfy (18) and (21), with $\mu>0$ given by (22), we obtain (25) with $Y_{2}\left(t, X_{t}\right)=f_{2}(X(t))$. We bound the second term on the righthand side of (25) as in (25). As to the third term, which is now

$$
\mathbf{E}\left[\max _{a_{n} \leq t \leq a_{n+1}}\left\|\int_{a_{n}}^{t} f_{2}(X(s)) d B(s)\right\|_{2}^{p}\right]
$$

by using the Burkholder-Davis-Gundy inequality, (9), the continuity of the solution, and (22), we obtain

$$
\begin{aligned}
\mathbf{E}\left[\max _{a_{n} \leq t \leq a_{n+1}}\left\|\int_{a_{n}}^{t} f_{2}(X(s)) d B(s)\right\|_{2}^{p}\right] \\
\quad \leq C_{p} \mathbf{E}\left[\left(\int_{a_{n}}^{a_{n+1}}\left\|f_{2}(X(s))\right\|_{F(d, r)}^{2} d s\right)^{\frac{p}{2}}\right] \\
\leq C_{p} \lambda^{p} \mathbf{E}\left[\left(\int_{a_{n}}^{a_{n+1}}\|X(s)\|_{2}^{2} d s\right)^{\frac{p}{2}}\right] \\
\leq C_{p} \lambda^{p} \mathbf{E}\left[\left(\left(a_{n+1}-a_{n}\right) \max _{a_{n} \leq t \leq a_{n+1}}\|X(t)\|_{2}^{2}\right)^{\frac{p}{2}}\right]
\end{aligned}
$$




$$
\begin{aligned}
& \leq C_{p} \lambda^{p} \mu^{\frac{p}{2}} \mathbf{E}\left[\max _{a_{n} \leq t \leq a_{n+1}}\|X(t)\|_{2}^{p}\right] \\
& \leq \frac{1}{3^{p-1}} \frac{1}{2} \mathbf{E}\left[\max _{a_{n} \leq t \leq a_{n+1}}\|X(t)\|_{2}^{p}\right] .
\end{aligned}
$$

Putting (27) and (33) into (25) gives

$$
\begin{aligned}
\frac{1}{2} \mathbf{E}\left[\max _{a_{n} \leq t \leq a_{n+1}}\|X(t)\|_{2}^{p}\right] \leq 3^{p-1} & \left\{\mathbf { E } \left[\left\|X\left(a_{n}\right)\right\|_{2}^{p}\right.\right. \\
& +\lambda^{p} \mu^{p-1} 2^{p-1} \int_{a_{n}}^{a_{n+1}} \mathbf{E}\left[\|X(s)\|_{2}^{p}\right] d s \\
& +\lambda^{p} \mu^{p-1} 2^{p-1}\left(\left\|K_{1}\right\|_{2}^{L^{1}\left(\mathbf{R}^{+}\right)}\right)^{p-1} \\
& \left.\times \int_{a_{n}}^{a_{n+1}}\left(\left\|K_{1}\right\|_{2} * \mathbf{E}\left[\|X\|_{2}^{p}\right]\right)(s) d s\right\} .
\end{aligned}
$$

From this inequality (31) can be easily inferred. The remainder of the proof of this theorem follows verbatim the line of argument in Theorem 4 after the inequality (31), and is therefore omitted.

Note that Theorem 5 has a direct deterministic counterpart. For the Volterra integrodifferential equation

$$
x^{\prime}(t)=f_{1}(x(t))+\int_{0}^{t} K_{1}(t-s) g_{1}(x(s)) d s
$$

it is true that $x(t) \in L^{p}\left(\mathbf{R}^{+}\right)$for $p \geq 1$ implies $x(t) \rightarrow 0$ as $t \rightarrow \infty$.

4. Examples. We illustrate Theorems 4 and 5 with some examples.

4.1 Scalar equation without instantaneous negative feedback. Our first example is an adaptation of a result in [10, Chapter 10], which is concerned with the boundedness of the moments of a scalar SFDE with bounded delay, but without requiring instantaneous negative feedback. Here, we modify the problem and functional so that we can study the convergence of solutions for the corresponding Itô-Volterra equation, which has unbounded delay. 
Let $X(t)$ be the unique strong solution of the SFDE given by

$$
d X(t)=\left(-\int_{0}^{t} k_{0}(t-s) X(s)+a_{1}\left(t, X_{t}\right)\right) d t+a_{2}\left(t, X_{t}\right) d B(t)
$$

where $B=\{B(t) ; 0 \leq t \leq 0\}$ is a standard one-dimensional Brownian motion. Let $k_{0} \in L^{1}\left(\mathbf{R}^{+}\right) \cap C\left(\mathbf{R}^{+} ; \mathbf{R}\right)$. Let $a_{1}, a_{2}:[0, \infty) \times$ $C\left(\mathbf{R}^{+} ; \mathbf{R}\right) \mapsto \mathbf{R}$ satisfy

$$
a_{j}\left(t, x_{t}\right)^{2} \leq \sigma_{j}^{2} x(t)^{2}+\int_{0}^{t} k_{j}(t-s) x(s)^{2} d s, \quad j=1,2,
$$

where $\sigma_{1}, \sigma_{2} \geq 0$ and $k_{1}, k_{2} \in L^{1}\left(\mathbf{R}^{+}\right) \cap C\left(\mathbf{R}^{+} ; \mathbf{R}^{+}\right)$. The equation can serve as a one-dimensional stochastic model in viscoelasticity where the solution is the strain, and $k_{0}$ is the relaxation function. We assume that $\int_{0}^{\infty} k_{0}(u) d u>0, \int_{0}^{\infty} u\left|k_{0}(u)\right| d u<1$, and

$$
\begin{gathered}
\int_{0}^{\infty} k_{0}(u) d u\left(1-\int_{0}^{\infty} u\left|k_{0}(u)\right| d u\right)-\frac{1}{2}\left(\sigma_{2}^{2}+\int_{0}^{\infty} k_{2}(u) d u\right) \\
-\left(\sigma_{1}^{2}+\int_{0}^{\infty} k_{1}(u) d u\right)^{1 / 2}\left(1+\int_{0}^{\infty} u\left|k_{0}(u)\right| d u\right)>0 .
\end{gathered}
$$

Assume that $a_{1}\left(t, x_{t}\right) \neq 0$, so $\sigma_{1}^{2}+\int_{0}^{\infty} k_{1}(s) d s>0$, and define

$$
\begin{aligned}
V\left(t, x_{t}\right)= & \left(x(t)-\int_{0}^{t} \int_{t}^{\infty} k_{0}(\tau-s) d \tau x(s) d s\right)^{2} \\
& +c_{1} \int_{0}^{t} \int_{t}^{\infty} \int_{v-s}^{\infty}\left|k_{0}(u)\right| d u d v x(s)^{2} d s \\
& +\int_{0}^{t} \int_{t}^{\infty}\left(k_{2}(\tau-s)+c_{2} k_{1}(\tau-s)\right) d \tau x(s)^{2} d s,
\end{aligned}
$$

where $c_{1}, c_{2}$ are nonnegative constants to be determined later. Thus

$$
\begin{aligned}
d V\left(t, X_{t}\right)= & \left\{2\left(X(t)-\int_{0}^{t} \int_{t}^{\infty} k_{0}(\tau-s) d \tau X(s) d s\right)\right. \\
& \times\left(a_{1}\left(t, X_{t}\right)-\int_{0}^{\infty} k_{0}(s) d s X(t)\right)+a_{2}\left(t, X_{t}\right)^{2}
\end{aligned}
$$




$$
\begin{aligned}
& +X(t)^{2}\left(c_{1} \int_{0}^{\infty} u\left|k_{0}(u)\right| d u+\int_{0}^{\infty} k_{2}(s)+c_{2} k_{1}(s) d s\right) \\
& \left.-c_{1} \int_{0}^{t} \int_{t-s}^{\infty}\left|k_{0}(u)\right| d u X(s)^{2} d s-\left(\left(k_{2}+c_{2} k_{1}\right) * X^{2}\right)(t)\right\} d t \\
& +2\left(X(t)-\int_{0}^{t} \int_{t}^{\infty} k_{0}(\tau-s) d \tau X(s) d s\right) a_{2}\left(t, X_{t}\right) d B(t) .
\end{aligned}
$$

For all $\gamma_{1}, \gamma_{2}, \gamma_{3}>0$, we have

$$
\begin{gathered}
\left|2 X(t) a_{1}\left(t, X_{t}\right)\right| \leq \frac{1}{\gamma_{1}} X(t)^{2}+\gamma_{1}\left(\sigma_{1}^{2} X(t)^{2}+\left(k_{1} * X^{2}\right)(t)\right), \\
\left|\gamma_{2} \int_{0}^{t} \int_{t-s}^{\infty}\right| k_{0}(u)|d u X(s) X(t) d s| \\
\leq \gamma_{2} \int_{0}^{t} \int_{t-s}^{\infty}\left|k_{0}(u)\right| d u X(s)^{2} d s+\frac{1}{\gamma_{2}} X(t)^{2} \int_{0}^{\infty} u\left|k_{0}(u)\right| d u,
\end{gathered}
$$

and

$$
\begin{aligned}
\mid 2 \int_{0}^{t} \int_{t}^{\infty} k_{0}(\tau-s) & d \tau X(s) d s a_{1}\left(t, X_{t}\right) \mid \\
\leq & \frac{1}{\gamma_{3}} \int_{0}^{t} \int_{t-s}^{\infty}\left|k_{0}(u)\right| d u X(s)^{2} d s \\
& +\gamma_{3} \int_{0}^{\infty} u\left|k_{0}(u)\right| d u\left(\sigma_{1}^{2} X(t)^{2}+\left(k_{1} * X^{2}\right)(t)\right) .
\end{aligned}
$$

Now, define $K_{j}=\int_{0}^{\infty} k_{j}(s) d s, K_{0}^{\prime}=\int_{0}^{\infty} u\left|k_{0}(u)\right| d u$, and set $c_{1}=$ $\left(1 / \gamma_{3}\right)+K_{0} \gamma_{2}, c_{2}=\gamma_{1}+\gamma_{3} K_{0}^{\prime}$. Putting the above estimates into (36), and using the notation just introduced yields, for $t \geq s \geq 0$ :

$$
\begin{aligned}
& V\left(t, X_{t}\right)-V\left(s, X_{s}\right) \\
& \leq \int_{s}^{t}\left(-2 K_{0}+\frac{1}{\gamma_{1}}+K_{0} \frac{1}{\gamma_{2}} K_{0}^{\prime}+c_{1} K_{0}^{\prime}+c_{2} K_{1}+\gamma_{1} \sigma_{1}^{2}+\gamma_{3} \sigma_{1}^{2} K_{0}^{\prime}\right. \\
& \left.\quad+K_{2}+\sigma_{2}^{2}\right) X(u)^{2}+\left(a_{2}\left(u, X_{u}\right)^{2}-\sigma_{2}^{2} X(u)^{2}-\left(k_{2} * X^{2}\right)(u)\right) d u \\
& \quad+\int_{s}^{t} 2\left(X(u)-\int_{0}^{u} \int_{u}^{\infty} k_{0}(\tau-v) d \tau X(v) d v\right) a_{2}\left(u, X_{u}\right) d B(u) .
\end{aligned}
$$


By putting $\gamma_{2}=1, \gamma_{1}=\gamma_{3}=\left(\sigma_{1}^{2}+K_{1}\right)^{-1 / 2}$, and

$$
A=2\left(K_{0}\left(1-K_{0}^{\prime}\right)-\frac{1}{2}\left(\sigma_{2}^{2}+K_{2}\right)-\sqrt{\sigma_{1}^{2}+K_{1}}\left(1+K_{0}^{\prime}\right)\right),
$$

(35) gives $A>0$. Moreover, the last inequality gives

$$
\mathbf{E}\left[V\left(t, X_{t}\right)\right]-\mathbf{E}\left[V\left(s, X_{s}\right)\right] \leq-A \int_{s}^{t} \mathbf{E}\left[X(u)^{2}\right] d u,
$$

provided $\mathbf{E}\left[X(0)^{4}\right]<\infty$. Since $V$ is nonnegative, $\mathbf{E}\left[X^{2}\right] \in L^{2}\left(\mathbf{R}^{+}\right)$, and by Theorem 4 , we have $\lim _{t \rightarrow \infty} \mathbf{E}\left[X(t)^{2}\right]=0$, along with $X \in L^{2}\left(\mathbf{R}^{+}\right)$ and $\lim _{t \rightarrow \infty} X(t)=0$, a.s. The calculations are more straightforward in the case when $a_{1}\left(t, x_{t}\right) \equiv 0$, but again (35) suffices to prove $\int_{0}^{\infty} \mathbf{E}\left[X(t)^{2}\right] d t<\infty$, with the same functional $V$ as before, where on this occasion, $c_{2}=0$.

4.2 Nondestabilization of scalar Volterra equation by noise. Our second example indicates an application of the extra sharpness obtained in Theorem 5. Consider the scalar linear Itô-Volterra equation

$$
d X(t)=\left(-a X(t)+\int_{0}^{t} k(t-s) X(s) d s\right) d t+\sigma X(t) d B(t)
$$

where $k \in L^{1}\left(\mathbf{R}^{+}\right) \cap C\left(\mathbf{R}^{+} ; \mathbf{R}^{+}\right)$, is strictly positive. Also consider the deterministic counterpart to (37) viz.,

$$
x^{\prime}(t)=-a x(t)+\int_{0}^{t} k(t-s) x(s) d s .
$$

According to Miller [14], the zero solution of (38) is uniformly asymptotically stable if and only all solutions of (38) are in $L^{1}\left(\mathbf{R}^{+}\right)$. Furthermore, all solutions are integrable if and only if $\lambda+a-\hat{k}(\lambda) \neq 0$, for all $\lambda \in \mathbf{C}$ with $\Re e(\lambda) \geq 0$ where $\hat{k}$ is the Laplace transform of $k$, refer to Grossman and Miller [7]. It is a consequence of these results, and work of Burton and Mahfoud [6] that solutions of the initial value problem (38) are integrable if and only if

$$
a>\int_{0}^{\infty} k(s) d s .
$$


Therefore, if we can show that $x(t)=\mathbf{E}[|X(t)|]$ is a solution of (38) with $x(0)=\mathbf{E}[|X(0)|]$, we will have established the following stability result.

Theorem 6. Suppose that $k$ is a strictly positive, continuous and integrable function. If the zero solution of (38) is uniformly asymptotically stable, then the solution of (37) satisfies $\mathbf{E}[|X|] \in L^{1}\left(\mathbf{R}^{+}\right)$, $\lim _{t \rightarrow \infty} \mathbf{E}[|X(t)|]=0$, and $X \in L^{1}\left(\mathbf{R}^{+}\right), \lim _{t \rightarrow \infty} X(t)=0$ a.s.

Proof. For (37), it has been shown in [3] that $X(t) / X(0)>0$ for all $t \geq 0$, a.s., and if $X_{1}(t)$ is the solution of (37) with initial condition 1 , then $X(t)=X_{1}(t) X(0)$. As $X(0)$ is independent of the process $X_{1}$, we have

$$
\mathbf{E}[|X(t)|]=\mathbf{E}\left[\left|X_{1}(t)\right|\right] \mathbf{E}[|X(0)|]=\mathbf{E}\left[X_{1}(t)\right] \mathbf{E}[|X(0)|] .
$$

But $x_{1}(t)=\mathbf{E}\left[X_{1}(t)\right]$ is a solution of (38) with initial condition $x_{1}(0)=1$, so therefore $x(t)=\mathbf{E}[|X(t)|]$ solves (38) with initial condition $x(0)=\mathbf{E}[|X(0)|]$. By hypothesis, $x(t) \in L^{1}\left(\mathbf{R}^{+}\right)$, so $\mathbf{E}[|X(t)|] \in L^{1}\left(\mathbf{R}^{+}\right)$, and the conclusions of Theorem 5 hold, as (37) is in the same form as (32).

It is appropriate to say that the presence of noise does not destabilize the (asymptotically) stable zero solution of (38) for, if all solutions of this problem tend to zero as $t \rightarrow \infty$ and are integrable, then all solutions of the stochastic problem (37) tend to zero as $t \rightarrow \infty$ and are integrable, both in first mean, and almost surely.

Theorem 5 enables us to obtain a weaker sufficient condition for the almost sure stability of the solution of (37) than that which can be obtained by first proving that $\mathbf{E}\left[X^{2}\right] \in L^{1}\left(\mathbf{R}^{+}\right)$, and by then using Theorem 4. Indeed, availing of Theorem 5.4 in [1] , we see that whenever

$$
a>\int_{0}^{\infty} k(s) d s+\sigma^{2},
$$

we have $\mathbf{E}\left[X^{2}\right] \in L^{1}\left(\mathbf{R}^{+}\right)$, and so $\mathbf{E}\left[X(t)^{2}\right] \rightarrow 0$ as $t \rightarrow \infty, X \in L^{2}\left(\mathbf{R}^{+}\right)$ a.s. and $X(t) \rightarrow 0$ as $t \rightarrow \infty$ a.s. Clearly, (40) is a more conservative sufficient condition for the almost sure asymptotic stability and a.s. square integrability of the solution of (37) than (39). 
Theorem 6 also enables us to show that there are scalar Volterra equations whose solutions cannot be exponentially stable in $p$-th mean, for any $p \geq 1$. In particular such solutions cannot be exponentially stable in mean square, as claimed in the introduction. Suppose that $k$ is positive and integrable, and obeys (39). Then all solutions of (38) are integrable, and tend to zero as $t \rightarrow \infty$. However, according to Murakami, if $k$ also obeys

$$
\int_{0}^{\infty} k(t) e^{\varepsilon t} d t=\infty
$$

for every $\varepsilon>0$, then every nontrivial solution of (38) obeys

$$
\limsup _{t \rightarrow \infty}|x(t)| e^{\varepsilon t}=\infty
$$

for every $\varepsilon>0$. Since $t \mapsto \mathbf{E}[|X(t)|]$ is a solution of (38) with $x(0)=\mathbf{E}[|X(0)|]$, we have

$$
\limsup _{t \rightarrow \infty} \mathbf{E}[|X(t)|] e^{\varepsilon t}=\infty
$$

for every $\varepsilon>0$; moreover, by Liapunov's inequality, we have

$$
\limsup _{t \rightarrow \infty} \mathbf{E}\left[|X(t)|^{p}\right] e^{\varepsilon t}=\infty
$$

for every $\varepsilon>0$, and every $p \geq 1$.

5. Extensions. In this paper, we have concentrated our analysis on the autonomous convolution Volterra integrodifferential equation initial value problem. However, it is not difficult to see that one can recover very similar results for nonautonomous nonconvolution equations of the form

$$
\begin{aligned}
d X(t)= & \left(f_{1}(t, X(t))+\int_{0}^{t} g_{1}(s, t, X(s)) d s\right) d t \\
& +\left(f_{2}(t, X(t))+\int_{0}^{t} g_{2}(s, t, X(s)) d s\right) d B(t)
\end{aligned}
$$

For instance, suppose $f_{1}, f_{2}$ are globally linearly bounded and obey local Lipschitz conditions in the space coordinate, and are continuous 
in time. Also, suppose that $g_{1}, g_{2}$ are Lipschitz in the space coordinate, continuous in the time coordinates, while also obeying bounds of the form $\|g(s, t, x)\| \leq k(t-s)\|x\|$ for some scalar continuous and integrable function $k$. If $f_{1}(t, 0)=f_{2}(s, t, 0)=0$, and $g_{1}(t, 0)=g_{2}(s, t, 0)=0$, then the conclusions of Theorem 4 hold provided $\mathbf{E}\left[\|X\|^{p}\right] \in L^{1}\left(\mathbf{R}^{+}\right)$ for some $p \geq 2$. If $g_{2}(s, t, x) \equiv 0$, an analogue of Theorem 5 also holds.

Theorem 4 has a counterpart in the mean square case for the general stochastic functional differential equation

$$
d X(t)=f_{1}\left(t, X_{t}\right) d t+f_{2}\left(t, X_{t}\right) d B(t),
$$

even in the case where the equation has infinite delay. In this case for the continuous function $x$ (which belongs to the appropriate initial history space) $x_{t}=\{x(s): s \leq t\}$. We define $\psi$ to be the initial function, so $\psi$ is in some subspace of $C\left((-\infty, 0] ; \mathbf{R}^{d}\right)$, and

$$
X(t)=\psi(t), \quad t \leq 0 .
$$

Of course, to ensure that one can study stability, we require that $f_{1}(t, 0)=0, f_{2}(t, 0)=0$ for all $t \geq 0$. We refer the interested reader to Chapter 3.2.5 and Chapter 10.1.1 of [10] for a description of the appropriate setting for the initial function space, and assumptions on the functionals $f_{1}, f_{2}$ in order to assure existence and uniqueness of strong solutions on $[0, \infty)$.

Now consider the unbounded delay case, under the hypothesis that the functionals $f_{1}, f_{2}$ obey

$$
\left\|f_{j}\left(t, x_{t}\right)-f_{j}\left(t, y_{t}\right)\right\|_{2}^{2} \leq \int_{-\infty}^{0}\|x(t+\theta)-y(t+\theta)\|_{2}^{2} d r_{j}(\theta)
$$

for any $t \geq 0$ and continuous functions $x$ and $y$ defined on $\mathbf{R}$ whose restrictions to the negative half line are in $B C((-\infty, 0])$, and $r_{1}, r_{2}$ are nonnegative, bounded and nondecreasing functions of bounded variation. A careful inspection of the proof of Theorem 4 now reveals that if the initial function, which one can take to be deterministic, without loss of generality, is in $B C((-\infty, 0]) \cap L^{2}((-\infty, 0])$, and the solution obeys

$$
\int_{0}^{\infty} \mathbf{E}\left[\|X(t)\|^{2}\right] d t<\infty
$$


then all the conclusions of Theorem 4 hold, with $p=2$.

Acknowledgment. The author is grateful to the anonymous referees for their careful scrutiny of the paper and helpful comments which have improved the presentation of the results. It is also a pleasure to thank David Reynolds for motivating this problem, and Elayne Kelly for many interesting discussions on this topic.

\section{REFERENCES}

1. J.A.D. Appleby, Almost sure stability of Itô-Volterra equations, Stochastic Anal. Appl., submitted.

2. J.A.D. Appleby and D.W. Reynolds, On the non-exponential convergence of asymptotically stable solutions of linear scalar Volterra integro-differential equations, J. Integral Equations Appl. 14 (2002), 109-118.

3. - Non-exponential stability of scalar stochastic Volterra equations, Statist. Probab. Lett. 62 (2003), 35-343.

4. L. Arnold, E. Oeljeklaus and E. Pardoux, Almost sure and moment stability for linear Itô equations, in Liapunov exponents, Springer, Bremen, 1984.

5. M.A. Berger and V.J. Mizel, Volterra equations with Itô integrals, I, J. Integral Equations 2 (1980), 187-245.

6. T.A. Burton and W.E. Mahfoud, Stability condition for Volterra equations, Trans. Amer. Math. Soc. 279 (1983), 143-174.

7. S.I. Grossman and R.K. Miller, Nonlinear Volterra integrodifferential equations, J. Differential Equations 13 (1973), 551-566.

8. U.G. Haussman, Asymptotic stability of the linear Itô equation in infinite dimensions, J. Math. Anal. Appl. 65 (1978), 219-235.

9. K. Itô and M. Nisio, On stationary solutions of a stochastic differential equation, J. Math. Kyoto Univ. 4 (1964), 1-75.

10. V. Kolmanovskii and A. Myshkis, Introduction to the theory and applications of functional differential equations, in Mathematics and its applications, Kluwer Academic Publishers, Dordrecht, 1999.

11. X. Mao, Exponential stability of stochastic differential equations, in Pure and Applied Mathematics, Marcel Dekker, New York, 1994.

12. - Stochastic differential equations and applications, in Horwood Series in Mathematics and Applications, Horwood, Chichester, 1997.

13. - Stability of stochastic integro-differential equations, Stochastic Anal. Appl. 18 (2000), 1005-1017.

14. R.K. Miller, Asymptotic stability properties of linear Volterra integrodifferential equations, J. Differential Equations 10 (1971), 485-506.

15. V.J. Mizel and V. Trutzer, Stochastic heredity equations: Existence and asymptotic stability, J. Integral Equations 7 (1984), 1-72. 
16. S. Murakami, Exponential stability for fundamental solutions of some linear functional differential equations, in Proceedings of the International Symposium: Functional differential equations, World Scientific, Singapore, 1990.

17. S. Murakami, Exponential asymptotic stability of scalar linear Volterra equations, Differential Integral Equations 4 (1991), 519-525.

18. J. Zabczyk, On the stability of infinite-dimensional linear stochastic systems, in Probability theory, Z. Ciesislski, PWN, Warsaw, 1979.

Centre for Modelling with Differential Equations (CMDE), School of Mathematical Sciences, Dublin City University, Glasnevin, Dublin 9, IRELAND

E-mail address: john.appleby@dcu.ie 